\title{
Méthodes de compactage appliquées aux matériaux alluvionnaires
}

\author{
Nilton VALLE \\ Ingénieur du Département de Route de Santa Catarina (DER/SC) - Brésil \\ Doctorant, Université de Caen \\ Jean-Claude BLIVET \\ Ingénieur, LRPC de Rouen - CETE Normandie Centre \\ Matoren KHAY \\ Ingénieur, CER - CETE Normandie Centre \\ Philippe REIFFSTECK \\ Chargé de Recherche, LCPC - Paris \\ Résumé
}

La reconstitution d'éprouvettes de sol en laboratoire est nécessaire quand on étudie des matériaux pour l'utilisation en remblais routiers, dans les barrages et plus généralement quand le prélèvement d'éprouvettes du sol naturel est impossible. Le présent travail consiste à comparer à l'aide d'essais triaxiaux, quatre modalités de compactage d'un matériau écrêté à $25 \mathrm{~mm}$ et comparer les résultats avec des éprouvettes prélevées à la main sur un remblai de référence construit pour cette expérience. La résistance au cisaillement du sol est presque la même pour les quatre modalités de compactage mais pour le module tangent la variation des valeurs est très significative.

\section{Abstract}

Preparation of remoulded samples for laboratory testing is necessary when soil for earthworks and dams is studied and when in-situ sampling is unlikely. The work presented consists to compare triaxial tests on remoulded samples of a coarse grained soil passing the $25 \mathrm{~mm} \mathrm{NF}$ test sieve compacted in four different manners and those on samples trimmed in blocks excavated from a trial embankment specially erected for this study. The shear strength is very similar for the four compaction techniques but the tangent modulus present important variations.

\section{Introduction}

Les sols alluvionnaires grossiers comportent des particules de dimensions très différentes. La dimension des plus gros éléments peut atteindre deux cent à trois cent millimètres. Pour ce type de sol, la reconstitution des éprouvettes pour les essais de caractérisation en laboratoire pose le problème de l'influence du mode de compactage sur les caractéristiques mesurées. Cette communication présente les résultats d'une étude de l'influence de quatre modes de compactage sur les caractéristiques de l'essai triaxial : compactage quasi-statique, par vibrocompression, par marteau vibrant et par impact à la dame Proctor. 
Pour cette étude, le matériau, un tout venant naturel alluvionnaire $0 / 80 \mathrm{~mm}$ a été écrêté à $25 \mathrm{~mm}$. Les éprouvettes cylindriques sont de diamètre de $152 \mathrm{~mm}$ et d'élancement deux.

Les résultats des essais triaxiaux obtenus sont ensuite comparés avec ceux effectués sur des éprouvettes prélevées dans un remblai compacté par une plaque vibrante.

\section{Rappel bibliographique}

Le mode de compactage joue un rôle très important dans le comportement d'un matériau, principalement à cause de l'augmentation de la densité.

Dans ce sens LAMBE et WHITMAN (1976) ont montré que dans le cas des sables, les densités les plus élevées sont obtenues avec le compactage par vibration.

Les conclusions de FENG et VITTON (1997) vont dans le même sens. Le compactage par vibration conduit à des niveaux de densité sèche plus élevés que le compactage par impact (figure 1).
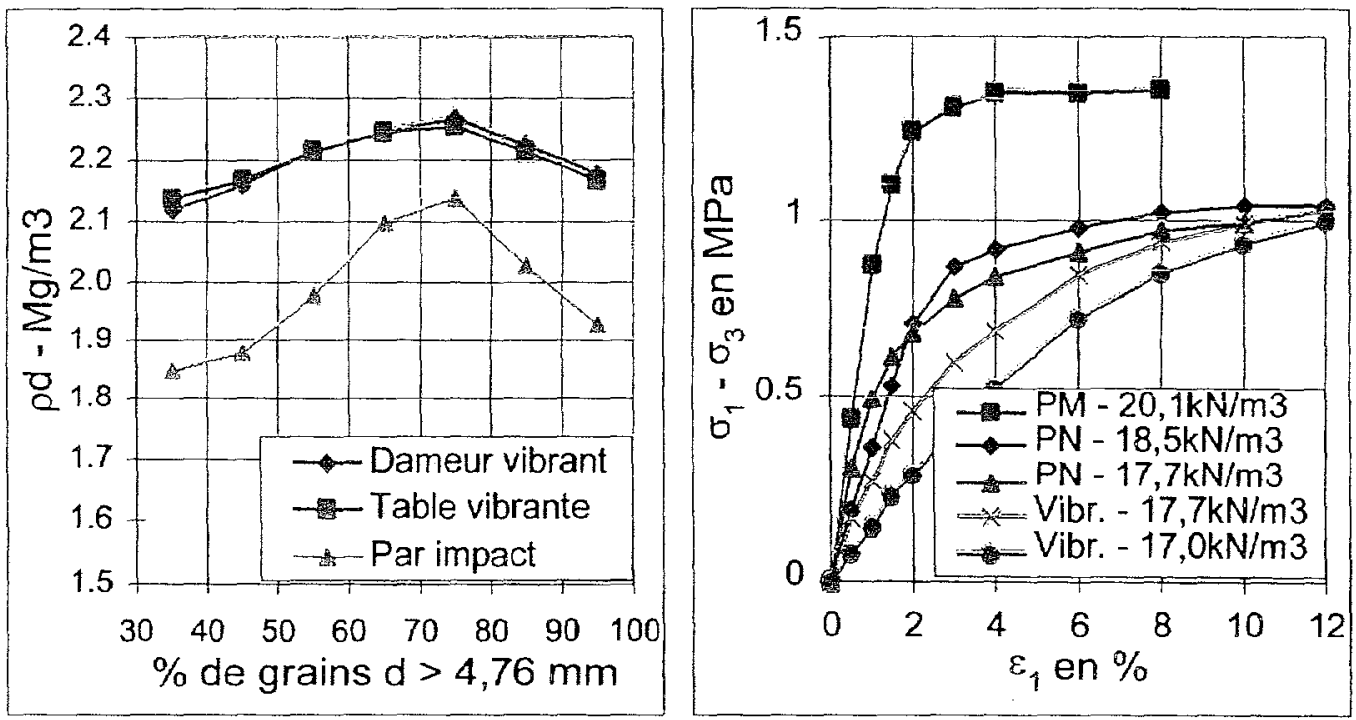

Figure 1: Influence du mode de compactage sur la masse volumique sèche d'un granite de $\operatorname{dmax}=60 \mathrm{~mm}$ (d'après FENG et VITTON, 1997) Figure 1: The effect of compaction methods on dry density
Figure 2 : Courbes contrainte-déformation d'une arène granitique pour différents modes de compactage $(\mathrm{PM}=$ Proctor Modifie et $\mathrm{PN}=$ Proctor Normal) (d'après KAST et BRAUNS, 1981)

Figure 2: Stress-strain behovior of granite fill samples with varying compaction 
Des essais sur un matériau d'arène granitique avec un $\mathrm{d}_{\max }=30 \mathrm{~mm}$ ont été effectués par KAST et BRAUNS (1981), dans un appareil triaxial de $200 \mathrm{~mm}$ de diamètre et de $200 \mathrm{~mm}$ de hauteur. Le matériau a été compacté par impact et par vibration. Ils ont vérifié que le comportement contrainte-déformation est très différent et dépendant du mode de compactage (figure 2). Selon les auteurs, cette différence s'explique par le fait que le compactage par impact donne une orientation des particules préférentielle et différente de celle du compactage vibrant.

Par ailleurs, CARVALHO et al. (1985) présentent des résultats où l'hétérogénéité d'échantillons de latérite compactés semi-statiquement affecte beaucoup les caractéristiques mécaniques des sols reconstitués.

Selon les auteurs, cette hétérogénéité résulte essentiellement du mode de compactage, en particulier à cause du frottement sol-moule. Ce même phénomène a été mis en évidence pour d'autres sols tels que les marnes.

\section{Description et caractéristiques du matériau}

Le matériau est tout venant de graves naturelles $0 / 80 \mathrm{~mm}$ provenant de dépôt alluvionnaire, silico-calcaire de la terrasse de la Vallée de Seine, extrait de la carrière de Criquebeuf sur Seine (Seine-Maritime).

Le tableau 1 donne les caractéristiques d'identification du matériau. Les courbes granulométriques de la grave $0 / 80 \mathrm{~mm}$ et des fractions écrêtées à 20 et 25 $\mathrm{mm}$ sont données à la figure 3 . Les caractéristiques Proctor sont celles des essais réalisés sur la fraction $0 / 20 \mathrm{~mm}$ et corrigées pour la fraction $0 / 25 \mathrm{~mm}$.

Tableau 1: Caractéristiques du matériau

Tableau 1: Physical and identification parameiers of the Criquebeuf soil

\begin{tabular}{|c|c|c|c|c|c|c|c|}
\hline & naturel & \multicolumn{3}{|c|}{ écrêté à $20 \mathrm{~mm}$} & \multicolumn{3}{|c|}{ écrêté à $25 \mathrm{~mm}$} \\
\hline $\mathrm{d}_{\max }(\mathrm{mm})$ & 80 & \multicolumn{3}{|l|}{20} & \multicolumn{3}{|l|}{25} \\
\hline$\%<80 \mu \mathrm{m}$ & 8,3 & \multicolumn{3}{|l|}{11,1} & \multicolumn{3}{|l|}{10,4} \\
\hline$\%<5 \mathrm{~mm}$ & 54,2 & \multicolumn{3}{|l|}{72,3} & \multicolumn{3}{|l|}{67,8} \\
\hline$\overline{\mathrm{C}_{\mathrm{U}}}$ & 80 & \multicolumn{3}{|l|}{26} & \multicolumn{3}{|l|}{32} \\
\hline $\mathrm{C}_{\mathrm{C}}$ & 0,17 & \multicolumn{3}{|l|}{0,83} & \multicolumn{3}{|l|}{0,58} \\
\hline VBS & 0,01 & \multicolumn{3}{|l|}{-} & \multicolumn{3}{|l|}{-} \\
\hline \multirow[t]{2}{*}{ Classification } & $\mathrm{D3}$ & & & & & & \\
\hline & & série 1 & série 2 & remblai & série 1 & série 2 & remblai \\
\hline$w_{\text {OPN }}(\%)$ & - & 10 & 9,3 & 9,2 & 9,3 & 8,7 & 8,6 \\
\hline$\rho_{\text {OPN }}\left(\mathrm{Mg} / \mathrm{m}^{3}\right)$ & - & 1,993 & 2,030 & 2,035 & 2,024 & 2,062 & 2,065 \\
\hline
\end{tabular}




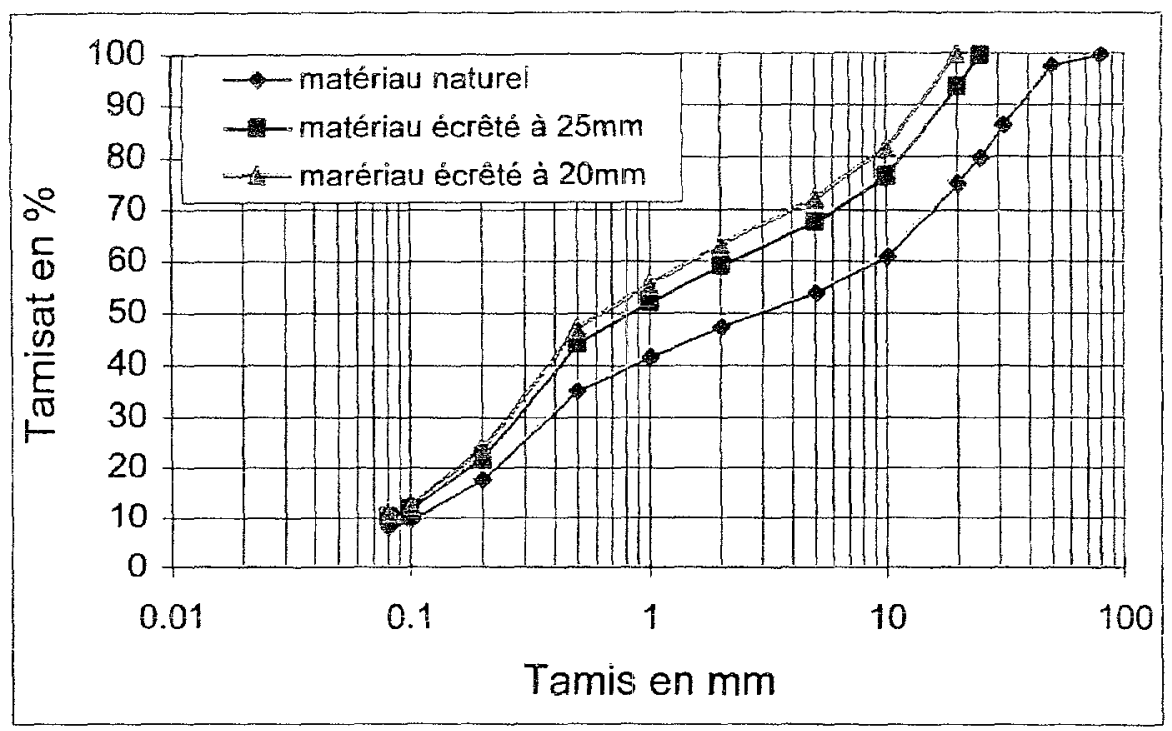

Figure 3 : Courbes granulométriques - (Criquebeuf sur Seine)

Figure 3 : Grading curves for Criquebeuf soil

\section{Description des méthodes de compactage}

Les différents protocoles de compactage en laboratoire et en place ont été définis de façon à se rapprocher des conditions de chantier.

\subsection{Compactage quasi-statique}

Pour le compactage quasi-statique, une presse de moulage des éprouvettes de laboratoire a été utilisée. L'éprouvette est compactée par un piston qui applique une charge verticaie de $40 \mathrm{kN}$ dans un moule métallique de $160 \mathrm{~mm}$ de diamètre et équipé d'un contre-moule intérieur en PVC de $152 \mathrm{~mm}$ de diamètre.

Une masse de matériau, de teneur en eau et masse volumique connues, a été introduite dans le moule de volume défini, qui contient une embase métallique (le disque d'espacement de l'essai Proctor). La procédure de compactage consiste à faire pénétrer le piston dans le moule qui s'appuie sur une embase métallique. Ensuite, le moule est renversé et, après on applique le piston de l'autre côté. Nous avons appliqué cette procédure jusquà la déformation maximale possible. Avec cette procédure, nous avons réussi à obtenir 97 à $98 \%$ de la masse volumique maximale de l'essai Proctor normal.

\subsection{Compactage par vibrocompression}

Nous avons utilisé un dispositif de vibrocompression pour confectionner en laboratoire des éprouvettes de densité et de teneur en eau prédéterminées. Nous avons utilisé un contre moule en $P V C$ à l'intérieur du moule pour arriver au diamètre désiré de $152 \mathrm{~mm}$. 
Une masse de matériau, de teneur en eau et masse volumique connue, a été introduite dans le moule de volume défini. Ce moule et le matériau qu'il contient subissent les effets :

- d'une compression simple dirigée selon l'axe du cylindre ;

- d'une vibration forcée dont la résultante multidirectionnelle est contenue dans un plan perpendiculaire à ce même axe. L'action combiné de cette compression et de cette vibration provoque un arrangement du sol qui conduit très rapidement aux compacités souhaitées.

\subsection{Compactage par marteau vibrant}

Nous avons utilisé un moule métallique (aluminium) de $152 \mathrm{~mm}$ de diamètre pour $304 \mathrm{~mm}$ de hauteur muni d'une rehausse métallique amovible.

Une membrane en latex (identique à celle de l'essai triaxial) est mise à l'intérieur du moule pour minimiser le frottement sol-moule. Une couche mince de graisse est interposée entre le moule et la membrane de façon à réduire le coefficient de frottement. Nous avons compacté le matériau en trois couches avec le marteau vibrant qui disposait d'une dame de compactage constituée d'un mouton cylindrique de $150 \mathrm{~mm}$ de diamètre. La charge est appliquée sur toute la surface de l'éprouvette et l'on suit l'opération à l'aide d'un dispositif de contrôle de hauteur pour chaque couche. La membrane de compactage a été enlevée pour la réalisation de l'essai triaxial.

\subsection{Compactage avec la dame Proctor}

Nous avons utilisé le même moule et la même façon de préparer l'éprouvette que pour le compactage par marteau vibrant ( $\S 4.3$.). Nous avons compacté le matériau en trois couches avec la dame dite "Dame Proctor Modifié " en donnant 56 coups à chaque couche. L'énergie de compactage correspond à celle de l'essai Proctor normai. Après le compactage, nous avons arasé l'excédent du matériau au niveau du moule et enlevé la membrane pour la réalisation de l'essai triaxial.

\subsection{Compactage du remblai}

Nous avons réalisé un remblai dont l'objectif est d'essayer de prélever des échantillons pour faire des éprouvettes pour les essais triaxiaux qui serviront de référence pour juger les protocoles appliqués en laboratoire. Nous avons réalisé le remblai de $1,00 \mathrm{~m} \times 1,00 \mathrm{~m}$ en trois couches de $20 \mathrm{~cm}$ compactées. Nous avons appliqué le protocole de compactage défini par le GTR afin d'atteindre l'objectif de densification q4. Pour chaque couche de $30 \mathrm{~cm}$ de matériau étalé, selon la GTR, nous avons réalisé dix passes d'une plaque vibrante de marque WACKERS classée PQ3.

Sur place, nous avons taillé à la main des éprouvettes avec l'aide d'un tube guide de $160 \mathrm{~mm}$ de diamètre et après au laboratoire, nous avons fini le travail en remplissant les trous avec le matériau 0-12,5 mm. Ensuite, nous avons mis le 
moule triaxial pour maintenir et conserver l'éprouvette jusqu'à la réalisation de l'essai (conservation dans une chambre climatisée).

\section{Présentation et analyse des résultats des essais triaxiaux}

Nous avons fait des essais triaxiaux consolidés sur des éprouvettes non saturées (Norme NF P 94-074). La consolidation isotrope a été faite pendant 15 heures avec l'application d'une contrainte correspondant à la contrainte radiale choisie $(50 \mathrm{kPa}$, ou $100 \mathrm{kPa}$, ou $150 \mathrm{kPa})$. La vitesse d'essai de cisaillement a été choisie à $0,1 \mathrm{~mm} / \mathrm{min}$., jusqu'à la déformation de $10 \%$. Pendant l'essai, nous n'avons mesuré que la déformation axiale et le déviateur de contrainte, une fois que la contrainte radiale a été maintenue constante.

Dans les tableaux 2 et 3 sont présentées les caractéristiques des éprouvettes avant la réalisation des essais triaxiaux, pour $\sigma_{3}=100 \mathrm{kPa}$. La $1^{\text {ire }}$ série et la $2^{\text {eme }}$ série d'essais portent sur le matériau de même origine mais légèrement différents, à cause de la variabilité naturelle du site.

Pour vérifier l'homogénéité de la masse volumique le long des éprouvettes, nous les avons auscultées au Banc gamma densimétrique avec des mesures à chaque centimètre suivant la génératrice. Ensuite, nous avons déterminé les teneurs en eau en divisant l'éprouvette en 6 parties de $5 \mathrm{~cm}$ chacune (figure 4). Nous voyons que la variation de la masse volumique est de sens contraire à celle de la teneur en eau.

Nous observons de manière évidente les zones intercouches avec des pics de densité, pour les éprouvettes a et b. Les densités sont plus élevées pour le "marteau vibrant" et le "Proctor" et plus faibles pour la vibrocompression.

Tableau 2: Caractéristiques des éprouvettes, pour $\sigma_{3}=100 \mathrm{kPa}-1^{\text {ere }}$ série

Table 2: Physical and geometrical parameters of the specimens - first set

\begin{tabular}{|c|c|c|c|c|c|c|c|c|c|}
\hline Mat & $\begin{array}{l}\text { Mode } \\
\text { Compactage }\end{array}$ & $\begin{array}{c}\mathrm{d} \\
(\mathrm{mm})\end{array}$ & $\begin{array}{c}\mathrm{h} \\
(\mathrm{mm})\end{array}$ & $\begin{array}{c}\rho_{\mathrm{OPN}} \\
\left(\mathrm{Mg} / \mathrm{m}^{3}\right)\end{array}$ & $\begin{array}{c}\rho_{\mathrm{d}} \\
\left(\mathrm{Mg} / \mathrm{m}^{3}\right)\end{array}$ & $\begin{array}{c}\mathrm{W} \\
(\%)\end{array}$ & $\begin{array}{c}\mathrm{TC}(*) \\
(\%)\end{array}$ & $\mathrm{e}$ & $\begin{array}{l}\mathrm{Sr} \\
(\%)\end{array}$ \\
\hline \multirow{7}{*}{$\begin{array}{l}\text { Crique- } \\
\text { beuf } \\
\text { sur } \\
\text { Seine } \\
\text { Ecrête } \\
0-25 \mathrm{~mm}\end{array}$} & Marteau 1 & 152 & 305 & 2,024 & 2,008 & 8,8 & 99,2 & 0,32 & 72,9 \\
\hline & \begin{tabular}{|l|} 
Vibro \\
\end{tabular} & 152 & 309 & 24 & 1,952 & 8,6 & 96,4 & 0,36 & 63,7 \\
\hline & Statique & 152 & 311 & 2,024 & 1,974 & 8 & 97,5 & 0,34 & 68,1 \\
\hline & Proctor & 152 & 305 & 2,024 & 1,971 & 8,7 & 97,4 & 0,34 & 66,9 \\
\hline & Proctor - M & 152 & 305 & 2,024 & 1,975 & 9 & 97,6 & 0,34 & 68,8 \\
\hline & Marteau 2 & 152 & 304 & 2,024 & 1,985 & 8,9 & 98,1 & 0,34 & 69,9 \\
\hline & Marteau - M & 152 & 306 & 2,024 & 1,971 & 9 , & 97,4 & 0,34 & 67,7 \\
\hline
\end{tabular}

$(*) \mathrm{TC}=\rho_{\mathrm{d}} / \rho_{\mathrm{OPN}}$ 
Tableau 3: Caractéristiques des éprouvettes, pour $\sigma_{3}=100 \mathrm{kPa}-2^{\mathrm{eme}}$ série

Table 3 : Physical and geometrical parameters of the specimens - second set

\begin{tabular}{|l|l|c|c|r|r|r|r|r|r|}
\hline Matériau & $\begin{array}{l}\text { Mode } \\
\text { Compactage }\end{array}$ & $\begin{array}{c}\mathrm{d} \\
(\mathrm{mm})\end{array}$ & $\begin{array}{c}\mathrm{h} \\
(\mathrm{mm})\end{array}$ & $\begin{array}{c}\rho_{\text {OPN }} \\
\left(\mathrm{Mg} / \mathrm{m}^{3}\right)\end{array}$ & $\begin{array}{c}\rho_{\mathrm{d}} \\
\left(\mathrm{Mg} / \mathrm{m}^{3}\right)\end{array}$ & $\begin{array}{c}\mathrm{w} \\
(\%)\end{array}$ & $\begin{array}{c}\mathrm{TC} \\
(\%)\end{array}$ & $\mathrm{e}$ & $\begin{array}{c}\mathrm{Sr} \\
(\%)\end{array}$ \\
\hline \multirow{2}{*}{$\begin{array}{l}\text { Crique- } \\
\text { beuf } \\
\text { sur }\end{array}$} & Marteau1-M & 152 & 305 & 2,062 & 2,034 & 7,3 & 98,6 & 0,30 & 63,9 \\
\cline { 2 - 10 } $\begin{array}{l}\text { Seine } \\
\text { Ecrête }\end{array}$ & Marteau 2-M & 152 & 305 & 2,062 & 2,021 & 7,9 & 98,0 & 0,31 & 67,3 \\
\cline { 2 - 10 } & Statique1 & 152 & 311 & 2,062 & 1,988 & 7,6 & 96,4 & 0,33 & 60,5 \\
\cline { 2 - 10 } & Statique2 & 152 & 309 & 2,062 & 2,012 & 7,6 & 97,6 & 0,32 & 63,5 \\
\cline { 2 - 10 } & Proctor1-M & 152 & 305 & 2,062 & 2,024 & 8,1 & 98,2 & 0,31 & 69,4 \\
\cline { 2 - 10 } & Proctor2-M & 152 & 305 & 2,062 & 2,024 & 7,5 & 98,2 & 0,31 & 64,3 \\
\cline { 2 - 10 } & Vibro1 & 152 & 304 & 2,062 & 2,037 & 7,3 & 98,8 & 0,30 & 64,3 \\
\cline { 2 - 10 } & Vibro2 & 152 & 312 & 2,062 & 1,998 & 6,7 & 96,9 & 0,33 & 54,4 \\
\cline { 2 - 9 } & Remblai1 & 156 & 308 & 2,065 & 1,937 & 8,6 & 93,8 & 0,37 & 61,9 \\
\cline { 2 - 9 } & Remblai2 & 156 & 306 & 2,065 & 1,963 & 9,0 & 95,1 & 0,35 & 68,1 \\
\hline
\end{tabular}
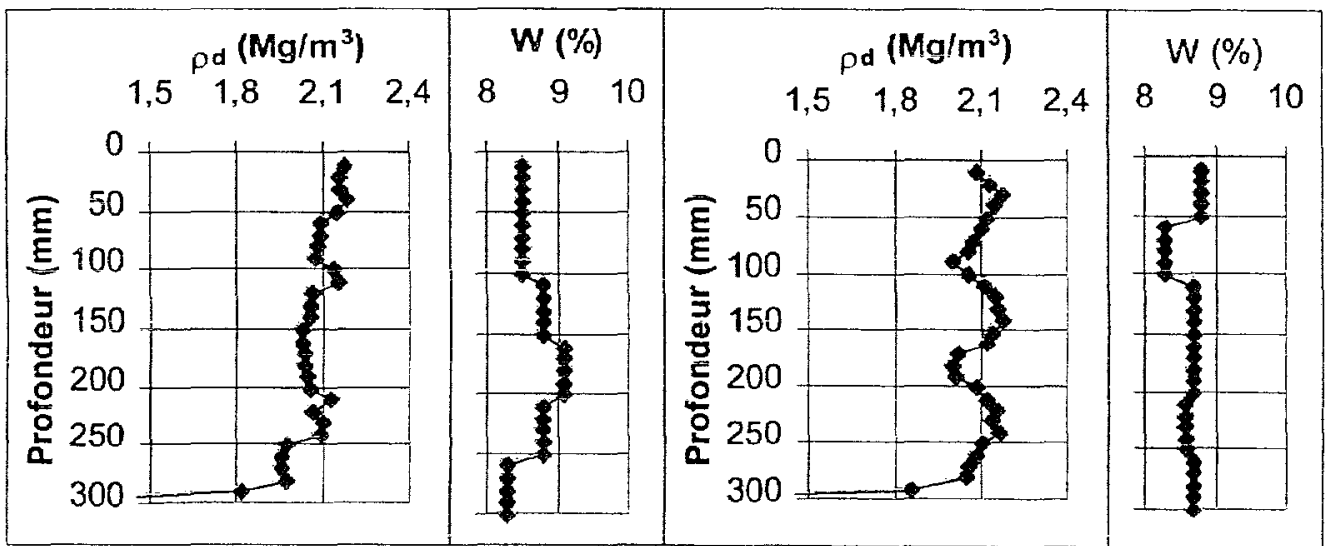

a) Marteau / hammer

b) Proctor / proctor
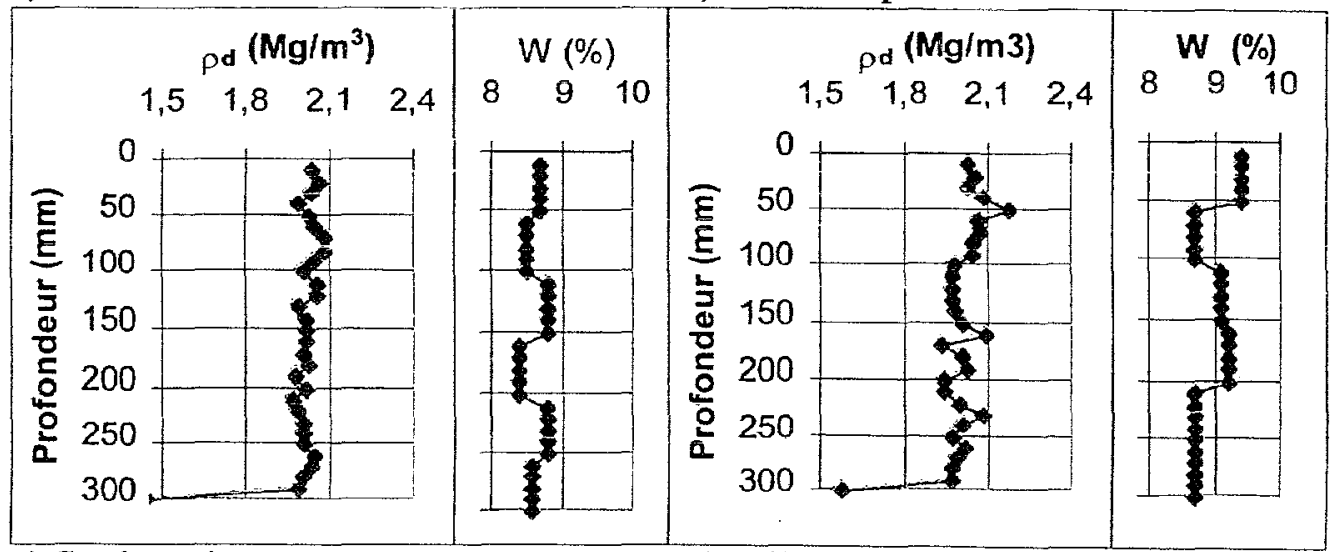

c) Statique / static

d) Vibrocompression/vibrocompaction

Figure 4 : Distribution de la masse volumique et de la teneur en eau dans les éprouvettes mesurées au Banc gamma

Figure 4: Repartition of dry density and moisture content in the specimen from gammadensitometer tests 
Les courbes contrainte-déformation sont présentées à la figure 5, pour chaque modalité de compactage.

Les courbes intrinsèques de la figure 6 montrent les résultats de la cohésion et de l'angle de frottement interne du matériau.

Les tableaux 4 et 5 présentent les résultats des essais triaxiaux. La $1^{\text {ere }}$ série et la $2^{\text {eme }}$ série d'essais portent sur le matériau de même origine mais légèrement différents, à cause de la variabilité naturelle du site.

D'après le tableau 4, les valeurs de la cohésion et de l'angle de frottement sont presque constantes, sauf pour le compactage Proctor et le remblai où la cohésion est plus faible et l'angle de frottement plus élevé. Ceci s'explique par la variation des caractéristiques du matériau pour le compactage Proctor et le remblai par rapport aux autres modalités.

Tableau 4: Résultats des essais, pour $\sigma_{3}=100 \mathrm{kPa}$ en condition de pic $-1^{\text {ere }}$ série

Table 4 : Test results for a confining pressure of $100 \mathrm{kPa}$ - first set

\begin{tabular}{|l|r|r|r|r|r|r|}
\hline Mode de Compactage & $\begin{array}{c}\left(\sigma_{1}-\sigma_{3}\right)_{\mathrm{f}} \\
(\mathrm{kPa})\end{array}$ & $\mathrm{c}(\mathrm{kPa})$ & $\begin{array}{c}\phi \text { en } \\
\text { degré }\end{array}$ & $\begin{array}{c}\delta \mathrm{h} / \mathrm{h} \\
(\%)\end{array}$ & $\begin{array}{c}\mathrm{E}_{\mathrm{tg}} \\
(\mathrm{MPa})\end{array}$ & $\begin{array}{c}\mathrm{E}_{\mathrm{tgmax}} \\
(\mathrm{MPa})\end{array}$ \\
\hline Marteau Vibrant 1 & 638 & 42,5 & 40,7 & 1,2 & 115,7 & 124,8 \\
\hline Marteau Vibrant 2 & 525 & $\mathbf{4 3 , 5}$ & 39,0 & 0,8 & 112,6 & $\mathbf{1 3 9 , 6}$ \\
\hline Marteau - Membrane & 531 & 43,8 & 39,0 & 1,1 & 125,8 & 125,8 \\
\hline Statique & 539 & 43,8 & 39,2 & 1,5 & 64,1 & $\mathbf{6 9 , 0}$ \\
\hline Vibrocompression & 501 & 39,7 & 37,8 & 1,0 & 116,0 & 147,8 \\
\hline Proctor & 473 & 19,8 & 41,2 & 2,2 & 79,7 & $\mathbf{8 7 , 5}$ \\
\hline Proctor - Membrane & 512 & 20,9 & 41,2 & 1,9 & 89,3 & 103,2 \\
\hline Remblai & & 13,4 & 42,0 & & & \\
\hline
\end{tabular}

Tableau 5: Résultats des essais, pour $\sigma_{3}=100 \mathrm{kPa}$ en condition de pic $-2^{\text {eme }}$ série

Table 5 : Test results for a confining pressure of $100 \mathrm{kPa}$ - second set

\begin{tabular}{|l|r|r|r|r|}
\hline Mode de Compactage & $\left(\sigma_{1}-\sigma_{3}\right)_{f}(\mathrm{kPa})$ & $\delta \mathrm{h} / \mathrm{h}(\%)$ & $\mathrm{E}_{\mathrm{t}}(\mathrm{MPa})$ & $\mathrm{E}_{\text {tomax }}(\mathrm{MPa})$ \\
\hline Marteau Vibrant1-M & 682 & 1,0 & 116,3 & 186,4 \\
\hline Marteau Vibrant2-M & 636 & 1,3 & 114,3 & 114,3 \\
\hline Statique1 & 455 & 1,3 & 66,9 & 75,5 \\
\hline Statique2 & 478 & 1,3 & 76,7 & 95,8 \\
\hline Vibrocompression1 & 608 & 2,2 & 88,3 & 105,1 \\
\hline Vibrocompression2 & 487 & 1,3 & 112,3 & 112,3 \\
\hline Proctor1-M & 489 & 2,7 & 53,4 & 53,4 \\
\hline Proctor2-M & 489 & 3,1 & 53,5 & 53,5 \\
\hline Remblai1 & 408 & 2,9 & 31,0 & 44,9 \\
\hline Remblai2 & 481 & 2,7 & 43,0 & 50,8 \\
\hline
\end{tabular}




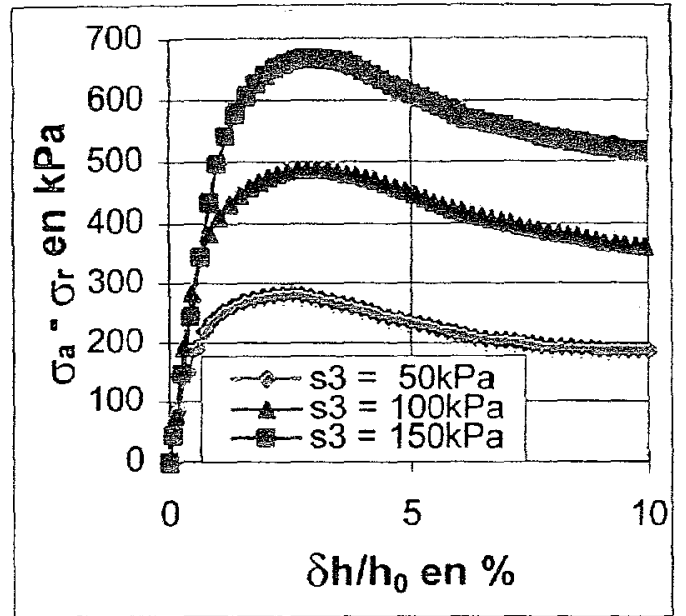

a) Proctor / proctor

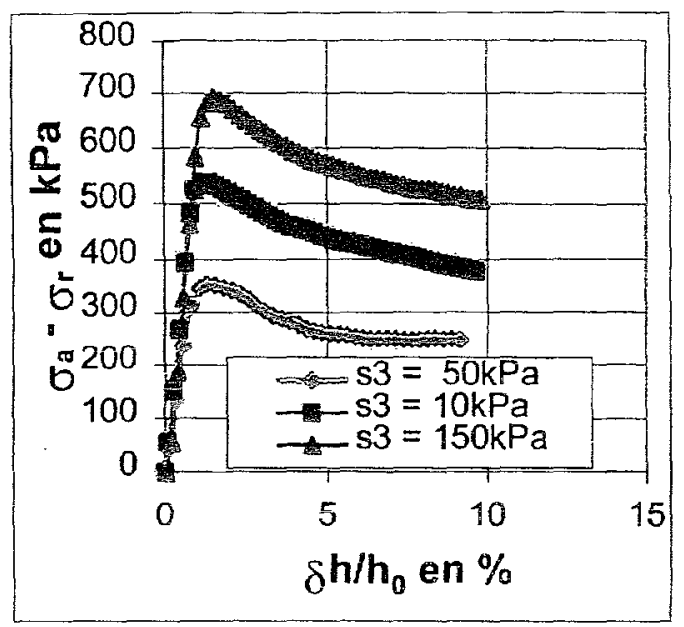

c) Statique / static

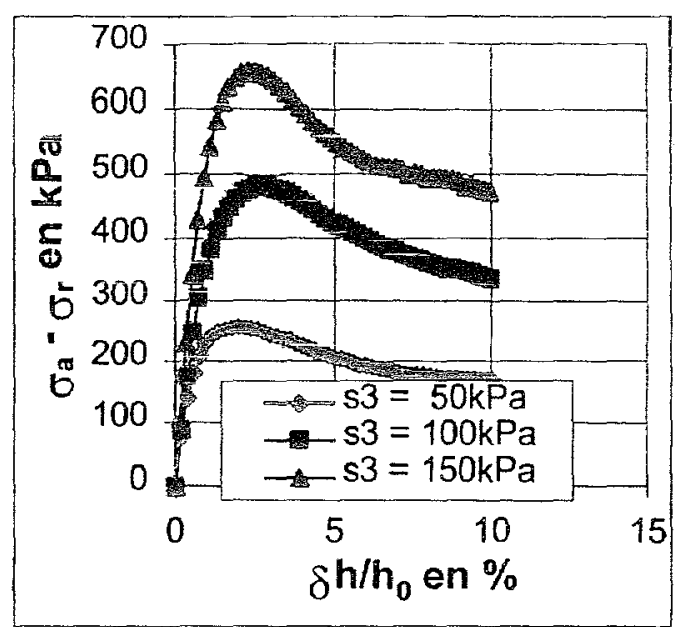

e) Petit Remblai

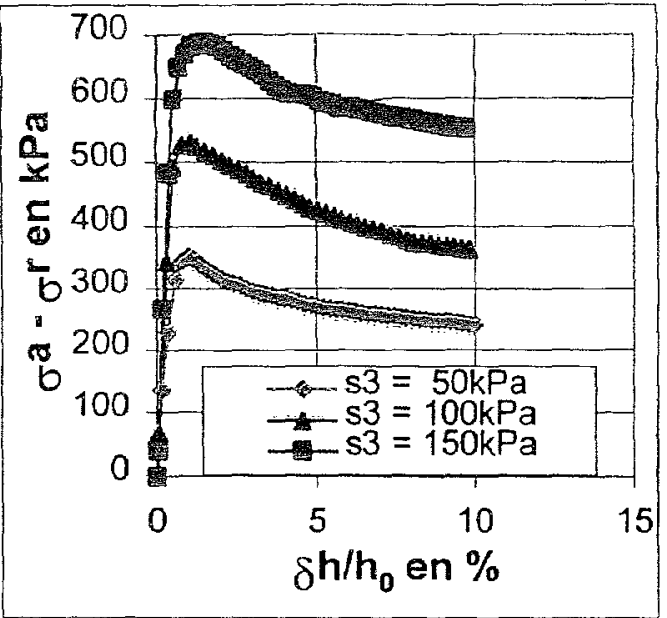

b) Marteau Vibrant / hammer

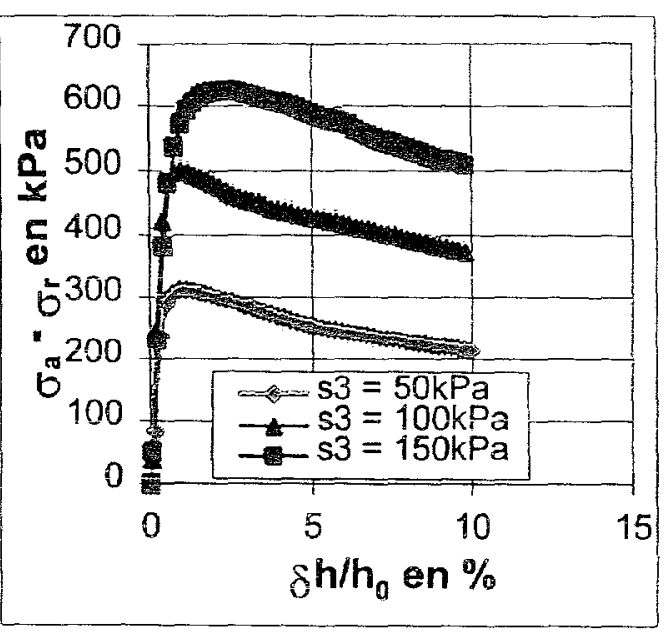

d) Vibrocompression / vibrocompaction

Figure 5: Courbes contrainte-déformation pour quatre compactages et le remblai

Figure 5 : Deviator stress versus axial strain for the four compaction techniques and embankment 

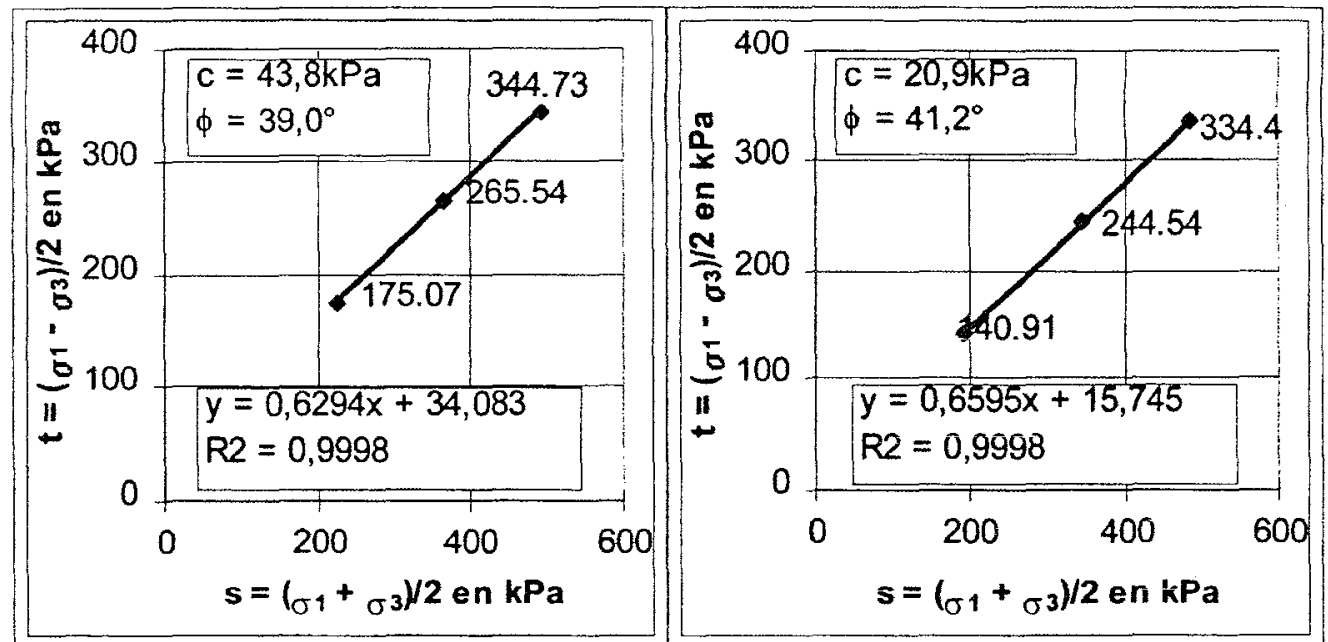

a)Marteau vibrant / hammer

b) Proctor / proctor
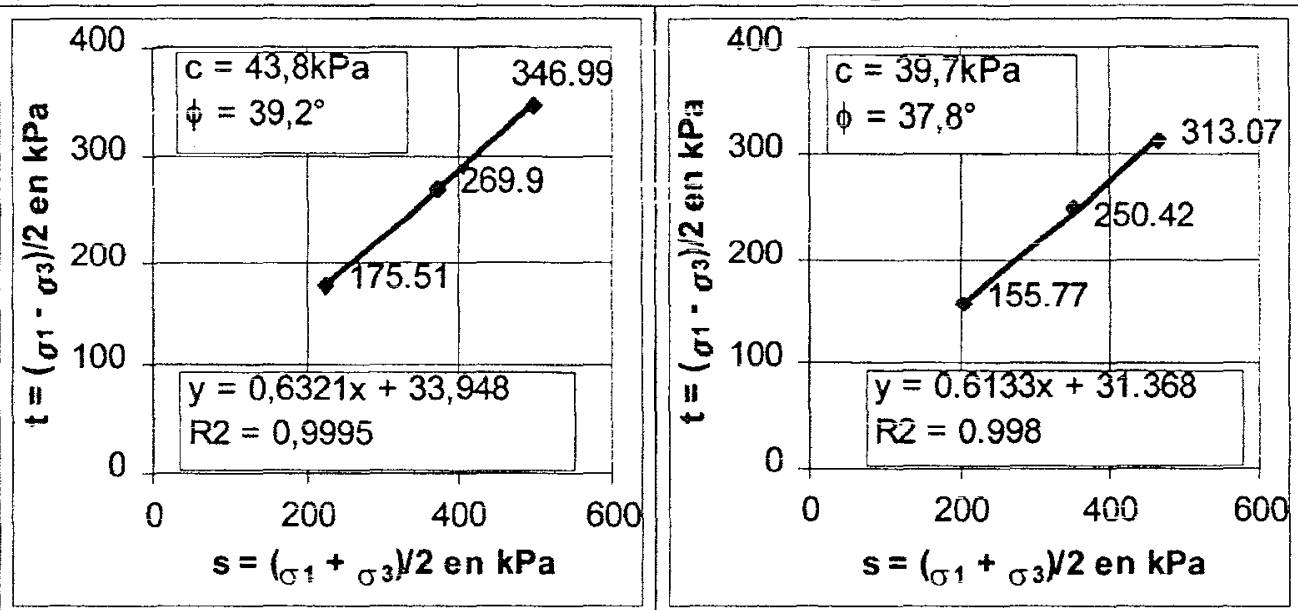

c)Statique / static

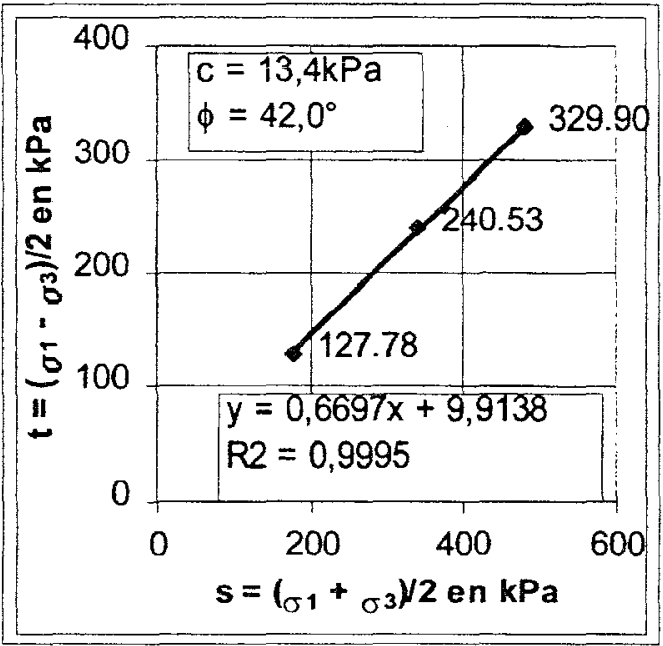

d) Vibrocompression / vibrocompaction

e)Petit Remblai / embankment

Figure 6: Courbes intrinsèques avec $c$ et $\phi$ - Eprouvettes des quatre compactages et du remblai

Figure $6:$ Failure envelope derived from triaxial tests for the four compaction techniques and the embankment 
Les courbes contrainte déformation pour les quatre modalités de compactage et pour le remblai sont présentées à la figure 7. Les courbes sont très proches, sauf pour le remblai où les pics de résistance ont des valeurs plus faibles et sont atteints pour des valeurs plus grande de la déformation. Les taux de compactage $\left(\mathrm{TC}=\rho_{\mathrm{d}} / \rho_{\mathrm{OPN}}\right)$ et la teneur en eau en sont sans doute la cause.

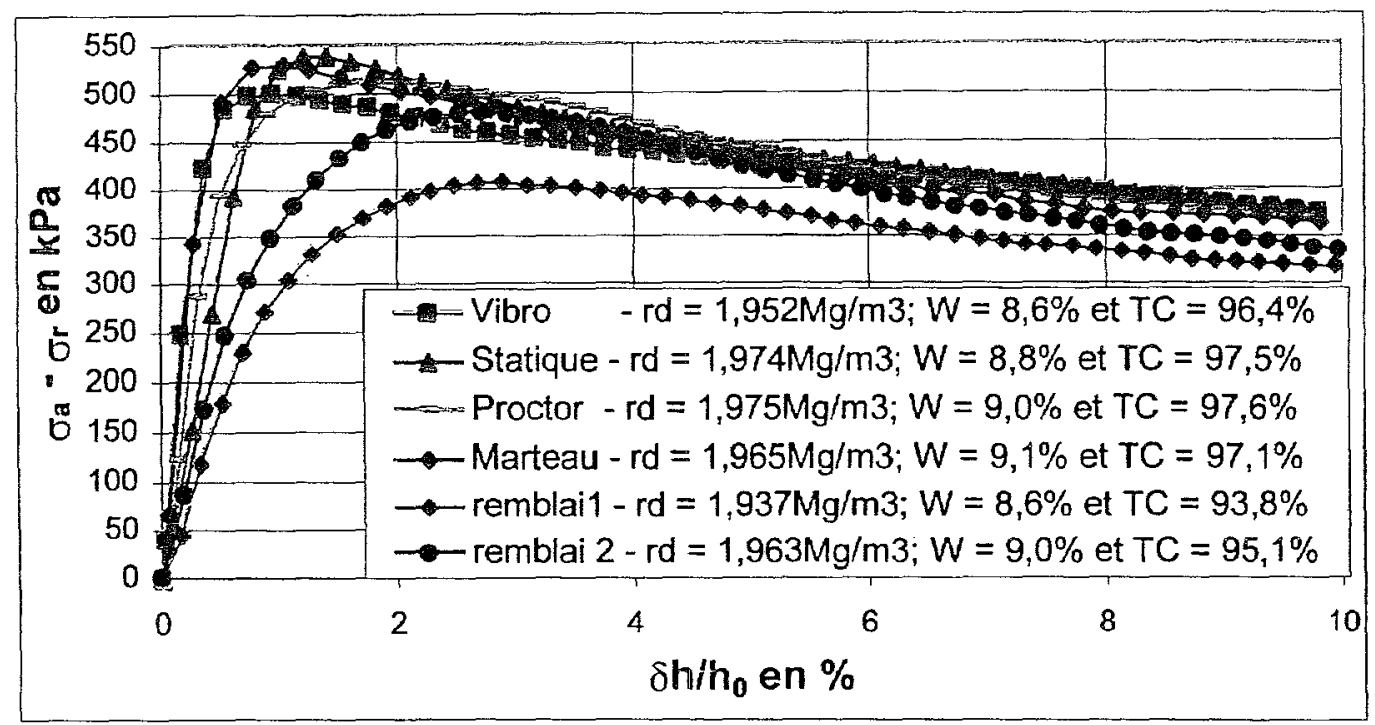

Figure 7 : courbe contrainte-déformation pour $\sigma_{3}=100 \mathrm{kPa} \quad\left(\mathrm{TC}=\rho_{\mathrm{d}} / \rho_{\mathrm{OPN}}\right)$ Figure 7 : Deviator stress versus axial strain for confining pressure of $100 \mathrm{kPa}$

Les valeurs calculées pour le module tangent, à $50 \%$ de la valeur de $\left(\sigma_{\mathrm{a}}-\sigma_{\mathrm{r}}\right)$, pour la contrainte radiale de $100 \mathrm{kPa}$ sont présentées au tableau 6 .

Tableau 6: Modules tangents, pour $\sigma_{3}=100 \mathrm{kPa}$ et pour $50 \%$ de $\left(\sigma_{\mathrm{a}}-\sigma_{\mathrm{z}}\right)$

Table ó: Tangent modulus for confining pressure of $100 \mathrm{kPa}$ and $50 \%$ of the deviatoric stress

\begin{tabular}{|l|r|r|r|r|r|}
\hline Compactage & $\begin{array}{c}\text { Statique } \\
(\mathrm{MPa})\end{array}$ & $\begin{array}{r}\text { Vibrocompression } \\
(\mathrm{MPa})\end{array}$ & $\begin{array}{r}\text { Marteau vibrant } \\
(\mathrm{MPa})\end{array}$ & $\begin{array}{c}\text { Proctor } \\
(\mathrm{MPa})\end{array}$ & $\begin{array}{r}\text { Remblai } \\
(\mathrm{MPa})\end{array}$ \\
\hline \multirow{2}{*}{$\begin{array}{l}\text { Valeurs } \\
\text { des modules } \\
\text { obtenus }\end{array}$} & 64,1 & 116,0 & 115,7 & 79,7 & 31,0 \\
\cline { 2 - 6 } & 66,9 & 88,3 & 112,6 & 89,3 & 43,0 \\
\cline { 2 - 6 } & 76,7 & 112,3 & 125,8 & 53,4 & \\
\cline { 2 - 6 } & & & 114,3 & 53,5 & \\
\hline Moyenne & 69,2 & & 116,3 & & 37,0 \\
\hline Ecart type & 6,6 & 105,5 & 116,9 & 68,9 & 8,5 \\
\hline CV & $9,5 \%$ & 15,0 & 5,1 & 18,3 & $22,9 \%$ \\
\hline
\end{tabular}

Le module tangent est le même pour le compactage Statique et pour le compactage Proctor d'une part, et, d'autre part, pour le compactage par Vibrocompression et le compactage au Marteau vibrant. Pour le remblai les valeurs des modules sont plus faibles, principalement à cause du plus faible taux de compactage et des teneurs en eau plus élevées. 
Les différences de module tangent suivant les modalités de compactage peuvent s'expliquer par des orientations différentes des particules pour chacune des modalités de compactage.

\section{Conclusion}

La résistance au cisaillement du sol étudié est peu affectée par le processus de confection des éprouvettes pour la réalisation des essais triaxiaux, même s'il y a des gradients de densité et de teneur en eau le long des éprouvettes.

Par contre, le module tangent est beaucoup plus sensible au mode de compactage des éprouvettes, les variations allant au delà du simple au double. Ceci montre que la façon de compacter un matériau agit d'une manière importante sur soñ comportement en déformation. Des phénomènes d'écrouissage ou de surconsolidation sont probablement en jeu dans ces différants modes de compactage.

\section{Remerciement}

Les auteurs tiennent à remercier le $\mathrm{CNPq}$ (Brésil) pour la bourse accordée à $\mathrm{N}$. VALLE, ce qui a permis la réalisation de cette recherche.

\section{Références Bibliographiques}

AFNOR (1994) Norme NF P 94-074. Essai à l'appareil triaxial de révolutionAppareillage, Préparation des éprouvettes, Essais UU, $C U+u, C D$.

BOURDEAU, Y. LAREAL, P. et MARCHAL, J. (1989). Résistance au cisaillement des alluvions du Rhône. Proceedings of the twelfth International Conference on Soil Mechanics and Foundation Engineering. Rio de Janeiro, volume I, p. 695-696.

CAMAPUM de CARVALHO, J, MIEUSSENS, $C$. et QUEIROZ de CARVALHO, J.B. (1985). Problèmes de reconstitution des éprouvettes de sol en laboratoire Proposition d'une méthode. Bulletin liaison des Laboratoires des Ponts et Chaussées $n^{\circ}$ 135, jan-févr, p. 77-83.

FENG, G. et VITTON, S.J. (1997). Laboratory Determination of compaction Criteria for Rockfill Material Embankments. Proceedings of the fourteenth International Conference on Soil Mechaniques and Foundation Engineering. Hamburg, volume 1, p. 485-88.

KAST, K. et BRAUNS, J. (1981). Dynamic compaction of rockfill samples. Proceedings of the tenth International Conference on Soil Mechaniques and Foundation Engineering. Stockholm, volume 1, p. 669-671.

LESLIE, D.D. (1963). Large-scale Triaxial Tests on Gravelly Soils. Proceeding of the second Panamerican Conference on Soil Mechanics and Foundation Engineering. Rio de Janeiro, volume 1, p. 181-202.

LAMBE, T.W. et WHITMAN, R.V. (1976). Mecánica de suelos. Editorial Limusa. Mexico, 582p.

SETRA-LCPC(1992). GTR. Réalisation des remblais et des couches de forme. 